\title{
Spectral properties of spontaneous photon emission by a material two-level system in a parabolic cavity
}

\author{
G. Alber ${ }^{1}$, A. V. Chizhov ${ }^{2,3}$ \\ ${ }^{1}$ Institut für Angewandte Physik, Technische Universität Darmstadt, \\ Darmstadt, 64289, Germany \\ ${ }^{2}$ Bogoliubov Laboratory of Theoretical Physics, Joint Institute for Nuclear Research, \\ Joliot-Curie, 6, Dubna, 141980, Russia \\ ${ }^{3}$ Dubna State University, Universitetskaya, 19, Dubna, 141980, Russia \\ chizhov@theor.jinr.ru
}

\begin{abstract}
The spectral properties of a photon spontaneously emitted by a material two-level system, modelling an atom or ion, in a parabolic cavity are investigated. In particular, we concentrate on the special case of a motionless two-level system positioned exactly at the focus of a parabolic cavity with a dipole moment oriented along the symmetry axis of this cavity. Treating the corresponding atom-field coupling in the dipoleand rotating wave approximation, it is demonstrated that inside the parabolic cavity the position and frequency dependence of the spectrum of the spontaneously emitted photon exhibits interesting interference patterns. These patterns are explored in detail with the help of a photon path representation of the first-order electric field correlation function. In the radiation, zone the spatial behavior of the spectrum reveals strong interference in particular at distances from the two-level system of the order of the focal length of the parabola. With increasing distances, these interference patterns decay except for an undepleted component surrounding the symmetry axis at an almost constant radius. Furthermore, the maximum of the frequency dependence of the spectrum exhibits a position-dependent frequency shift with respect to the atomic resonance frequency.
\end{abstract}

Keywords: cavity QED, spontaneous emission.

Received: 30 August 2017

Revised: 11 September 2017

\section{Introduction}

A material two-level system, modelling an atom or ion, interacting with the quantized radiation field is the paradigm of an elementary quantum optical system. Despite its simplicity, it exhibits many fundamental phenomena characteristic for quantum theory which also offer interesting possibilities for innovative quantum technological applications [1]. Within this scenario, the almost resonant exchange of energy between a single photon of the radiation field and a trapped motionless two-level system represents the simplest physically realizable model system. Particularly interesting phenomena occur in this simple model system if the photonic mode structure is engineered by the presence of a cavity which imposes additional physical boundary conditions.

In quantum electrodynamical experiments [2], manipulations of the photonic mode structure have already been pushed to the extreme case that approximately only a single mode of the electromagnetic radiation field is capable of exchanging energy with a trapped motionless atom almost resonantly. In these cases, which can be described within the Jaynes-Cummings-Paul model $[3,4]$, the cavity induced modification of the interaction between a two-level system and a photon can lead to vacuum Rabi oscillations or to collapse and revival phenomena [5].

A series of recent experiments [6] has started to explore a different dynamical regime in which a single approximately motionless two-level ion is trapped at the focus of a parabolic mirror. In view of the peculiar focusing properties of a parabolic mirror an intense exchange of energy between a single photon and the trapped material two-level system is expected in this scenario. In addition, this physical system offers interesting possibilities for applications in quantum communication [7], for example, as it allows for an almost lossless coupling in and coupling out of the electromagnetic field energy into and out of the cavity. Contrary to the case of extreme mode selection, in this scenario this half open cavity supports a continuum of photonic modes which can couple to the trapped material two-level system. This continuum of modes, however, is structured by the presence of the parabolic cavity so that interesting dynamical modifications take place, which range from modifications of the spontaneous decay rate of the trapped two-level system in comparison with the free-space scenario by the Purcell effect [8] to intricate recurrence effects observable in the time evolution of the trapped two-level system originating from photonic reflections at the boundary of the cavity $[9,10]$. 
In this paper we generalize previous investigations into the interaction of a trapped approximately motionless material two-level system with a single photon inside a parabolic cavity [10] and explore the spectral properties of a spontaneously emitted photon inside this cavity. For this purpose, we apply our recently developed classical path representation of photonic transition amplitudes to the description of the electric field correlation function of a single photon and to the corresponding spectrum. This position-dependent photonic correlation function and its corresponding spectrum produced by the trapped spontaneously decaying two-level system can be measured by positioning a photon detector with appropriate spectral properties at various positions inside the parabolic cavity. Our subsequent investigations demonstrate that in the radiation zone, the spatial behavior of the spectrum reveals strong interference effects. These interference effects reflect the intricate photonic dynamics inside the parabolic cavity in the presence of the trapped material two-level system which originates from the interference of probability amplitudes associated with different photon paths.

This paper is organized as follows: In Sec. 2, the quantum optical model of a trapped motionless two-level system interacting with the quantized radiation field inside a parabolic cavity is introduced and previous results are summarized briefly. Based on these previous results, characteristic dynamical properties of the spontaneous decay process and its influence on the first-order electric field correlation function of the radiation field are discussed in Sec. 3. Subsequently, the resulting spectrum of a photon spontaneously emitted by the two-level system trapped in the focus of the parabolic cavity is explored in Sec. 4.

\section{The quantum optical model}

In this section, we introduce the quantum optical model describing a two-level system positioned in the focus of a parabolic cavity and interacting with the quantized radiation field.

In particular, we consider a motionless material two-level system placed at a fixed position in a parabolic optical cavity with an ideally conducting wall. The Hamiltonian describing the dynamics of its interaction with the quantized radiation field is given by [10]:

$$
\hat{H}=\hat{H}_{A}+\hat{H}_{F}+\hat{H}_{A F}
$$

with the (free) Hamiltonian:

$$
\hat{H}_{A}=E_{e}|e\rangle\left\langle e\left|+E_{g}\right| g\right\rangle\langle g|,
$$

of the two-level system with its ground $|g\rangle$ and its excited state $|e\rangle$. These two states are assumed to be of opposite parity. The (free) dynamics of the quantized radiation field inside the parabolic cavity is described by the Hamiltonian:

$$
\hat{H}_{F}=\sum_{n} \int d \omega \hbar \omega \hat{a}_{\omega, n}^{\dagger} \hat{a}_{\omega, n} .
$$

The creation and destruction operators of the field modes are denoted by $\hat{a}_{\omega, n}^{\dagger}$ and $\hat{a}_{\omega, n}$. These modes are labeled by their continuous frequencies $\omega$ and by the discrete, integer-valued parameter $n$ originating from the boundary conditions imposed by the parabolic cavity. Consistent with the Coulomb gauge the corresponding orthonormal and frequency normalized mode functions $\mathbf{g}_{\omega, n}(\mathbf{x})$ fulfill the transversality condition $\nabla \cdot \mathbf{g}_{\omega, n}(\mathbf{x})=0$ and are solutions of the Helmholtz equation:

$$
\left(\nabla^{2}+\frac{\omega^{2}}{c^{2}}\right) \mathbf{g}_{\omega, n}(\mathbf{x})=0
$$

with boundary conditions of an ideally conducting parabolic mirror with focal length $f$. ( $c$ denotes the speed of light in vacuum.) The interaction between the two-level system positioned at $\mathbf{x}_{A}$ and the quantized radiation field is treated in the dipole- and rotating-wave approximations and is described by the Hamiltonian:

$$
\hat{H}_{A F}=-i \sum_{n} \int d \omega \sqrt{\frac{\hbar \omega}{2 \epsilon_{0}}} \mathbf{d} \cdot \mathbf{g}_{\omega, n}\left(\mathbf{x}_{A}\right) \hat{a}_{\omega, n}|e\rangle\langle g|+\text { h.c. },
$$

with the atomic dipole matrix element $\mathbf{d}=\langle e|\hat{\mathbf{d}}| g\rangle$ between the excited state $|e\rangle$ and the ground state $|g\rangle$.

In our subsequent discussion, we assume that the atom is located at the focus of the parabolic mirror, i.e. $\mathbf{x}_{A}=0$, and that its dipole moment is oriented along the symmetry axis of the parabolic cavity, i.e. $\mathbf{d}=d \mathbf{e}_{z}$. As a consequence, the mode functions $\mathbf{g}_{\omega, n}(\mathbf{x})$ solving Eq. (4) and capable of coupling to this atomic dipole can be written in the form [10]: 


$$
\mathbf{g}_{\omega, n}(\mathbf{x})=\nabla \times \mathbf{G}_{\omega, n}(\mathbf{x})
$$

with

$$
\begin{aligned}
\mathbf{G}_{\omega, n}(\mathbf{x}) & =\frac{1}{\sqrt{2 \pi \omega N_{\omega, n}}} \frac{\chi_{\omega, n}(\xi)}{\sqrt{\xi}} \frac{\chi_{\omega, n}(\eta)}{\sqrt{\eta}} \mathbf{e}_{\phi}, \\
\chi_{\omega, n}(\xi) & =\sqrt{\frac{4}{\pi k}} F_{0}\left(\alpha_{n} / k, k \xi / 2\right), \\
\chi_{\omega, n}(\eta) & =\sqrt{\frac{4}{\pi k}} F_{0}\left(-\alpha_{n} / k, k \eta / 2\right) .
\end{aligned}
$$

Thereby, we have introduced parabolic coordinates $(\xi, \eta, z)$ which are related to the corresponding symmetry adapted cylindrical coordinates $(\rho, \phi, z)$ by $\xi=r+z$ and $\eta=r-z$ with $r=\sqrt{\rho^{2}+z^{2}}$. The relevant mode functions involve the Coulomb functions $F_{L}(\mu, x)$ [11] in these parabolic coordinates with $L=0$ and with wave number $k=\omega / c$. The normalization factors appearing in Eq. (7) are given by:

$$
\mathscr{N}_{\omega, n}=\int_{0}^{2 f} d \eta \frac{\chi_{\omega, n}^{2}(\eta)}{\eta}
$$

The separation constants $\alpha_{n}$ are determined by the relevant boundary conditions at the surface of the ideally conducting parabola described by the equation $\eta=2 f$, i.e.:

$$
\left.\frac{d \chi_{\omega, n}(\eta)}{d \eta}\right|_{\eta=2 f}=0
$$

Eq. (9) ensures the absence of the tangential component of the electric-field operator $\hat{E}_{\xi}(\mathbf{x}) \equiv \mathbf{e}_{\xi} \cdot \hat{\mathbf{E}}(\mathbf{x})$ on the surface of the parabola, where:

$$
\hat{\mathbf{E}}(\mathbf{x})=i \sum_{n} \int d \omega \sqrt{\frac{\hbar \omega}{2 \epsilon_{0}}} \mathbf{g}_{\omega, n}(\mathbf{x}) \hat{a}_{\omega, n}+\text { h.c. } \equiv \hat{\mathbf{E}}^{+}(\mathbf{x})+\hat{\mathbf{E}}^{-}(\mathbf{x}) .
$$

\section{Photon path representation of the electric-field correlation function}

Based on the results of Sec. 2, we explore the first-order electric-field correlation function of a photon spontaneously emitted by the two-level system. For this purpose, we develop a classical photon path representation for the relevant one-photon transition amplitude in the radiation zone, i.e. at distances from the radiating two-level system large in comparison with the wave length of the spontaneously emitted photon. This classical photon path representation exhibits the intricate photonic dynamics inside the parabola with sequences of reflections at the boundary and elastic scatterings by the two-level system [9].

If the two-level system is initially in its excited state $|e\rangle$ without any photon in the radiation field, the subsequent time evolution of the quantum state of the atom-field system is of the general form:

$$
|\psi(t)\rangle=A_{e}(t)|e\rangle|0\rangle+\sum_{n} \int_{0}^{\infty} d \omega A_{\omega, n}(t)|g\rangle \hat{a}_{\omega, n}^{\dagger}|0\rangle .
$$

The time-dependent coefficients $A_{e}(t)$ and $A_{\omega, n}(t)$ fulfill the Schrödinger equation with Hamiltonian (1). In the long-time limit, i.e. $t \gg 1 / \Gamma_{0}$ with $\Gamma_{0}$ denoting the spontaneous decay rate of state $|e\rangle$, one obtains the result [10]:

$$
A_{\omega, n}(t)=i \sqrt{\frac{\omega}{2 \hbar \epsilon_{0}}} \mathrm{e}^{-i \omega t} \frac{\mathbf{d} \cdot \mathbf{g}_{\omega, n}\left(\mathbf{x}_{A}\right)}{\omega-\omega_{0}-i \Gamma_{0} / 2} .
$$

Information on the dynamics of the spontaneously emitted photon is contained in the first-order electric-field correlation function which is related to this probability amplitude by:

$$
\left\langle\psi\left(t_{1}\right)\left|\hat{\mathbf{E}}^{-}\left(\mathbf{x}_{1}\right) \otimes \hat{\mathbf{E}}^{+}\left(\mathbf{x}_{2}\right)\right| \psi\left(t_{2}\right)\right\rangle=\nabla \times \mathbf{F}^{*}\left(\mathbf{x}_{1}, t_{1}\right) \otimes \nabla \times \mathbf{F}\left(\mathbf{x}_{2}, t_{2}\right),
$$

with the effective one-photon amplitude: 


$$
\mathbf{F}(\mathbf{x}, t)=-i \sum_{n} \int_{0}^{\infty} d \omega \sqrt{\frac{\hbar \omega}{2 \epsilon_{0}}} A_{\omega, n}^{*}(t) \mathbf{G}_{\omega, n}(\mathbf{x}) .
$$

If the focal length $f$ of the parabolic cavity is not too large in the sense $c / \omega_{0} \ll f \ll c / \Gamma_{0}$, in the radiation zone this effective one-photon amplitude is given by [10]:

$$
\mathbf{F}(\mathbf{x}, t)=-i \mathbf{e}_{\phi} \frac{k_{0} d}{4 \pi \epsilon_{0} \rho} \sum_{M=0}^{\infty} \mathrm{e}^{-i 2 \pi M n_{0}} \mathrm{e}^{\left(i \omega_{0}-\Gamma_{0} / 2\right)(t-r / c)}\left[\frac{\theta(t-r / c-M T)}{\cosh \Lambda_{M}\left(\rho, z ; k_{0}\right)}-\frac{\theta(t-z / c-M T)}{\cosh \Theta_{M-1}\left(\rho, z ; k_{0}\right)}\right],
$$

with:

$$
\begin{aligned}
& \Lambda_{L}\left(\rho, z ; k_{0}\right)=\ln \left(\frac{r-z}{r+z}\right)+2 L \mathscr{S}\left(k_{0} f\right), \\
& \Theta_{L}\left(\rho, z ; k_{0}\right)=\ln \left(\frac{2 f}{\rho}\right)^{2}+2 L \mathscr{S}\left(k_{0} f\right),
\end{aligned}
$$

and with the diffraction function:

$$
\mathscr{S}(u)=\int_{0}^{u} d y \frac{\sin ^{2} y}{y} .
$$

In Eq. (15), the radiation zone refers to distances from the radiating two-level system which are large in comparison to the optical wave length characterizing the resonant atomic transition.

Eq. (15) represents the effective one-photon amplitude determining the electric-field correlation function as a sum of contributions $M$ which can be associated with repeated returns of the spontaneously emitted photon back to the atom. Thereby, this photon evolves periodically along a straight-line classical path extending along the symmetry axis of the parabolic cavity from its focal point, where the two-level system is positioned, to its boundary and back again. The time the photon requires for this motion is given by the period $T=2 \mathrm{f} / \mathrm{c}$ and $2 \pi n_{0}$ with $n_{0}=k_{0} f / \pi-1 / 2$ is the corresponding classical eikonal associated with this photon path. The integer $M$ enumerates the number of reflections of this photon path at the boundary of the parabola.

\section{Spectrum of the spontaneously emitted photon}

Based on the results of Sec. 3, we explore characteristic properties of the spectrum of the radiation field produced by the spontaneously emitted photon in the radiation zone, i.e. at distances from the radiating two-level system large in comparison with the wave length of the spontaneously emitted photon.

In general, the power spectrum of a photon spontaneously emitted by the material two-level system and detected at position $\mathbf{x}$ with frequency $\omega_{S}$ is related to the first-order electric-field correlation function of Eq. (13) by [12]:

$$
S\left(\mathbf{x}, \omega_{S}\right)=\int_{0}^{\infty} d t_{1} \int_{0}^{\infty} d t_{2} \mathrm{e}^{-i \omega_{S}\left(t_{1}-t_{2}\right)}\left\langle\psi\left(t_{1}\right)\left|\hat{\mathbf{E}}^{-}(\mathbf{x}) \cdot \hat{\mathbf{E}}^{+}(\mathbf{x})\right| \psi\left(t_{2}\right)\right\rangle .
$$

Performing the time integrations, we find from Eqs. (13) and (15) the final result, which is conveniently represented in the form:

$$
S\left(\mathbf{x}, \omega_{S}\right)=\left(\frac{\omega_{0}^{2} d}{4 \pi \epsilon_{0} c^{2}}\right)^{2} \cdot \frac{1}{\left(\omega_{0}-\omega_{S}\right)^{2}+\Gamma_{0}^{2} / 4} \cdot I_{\mathrm{mir}}\left(\rho, z ; \omega_{S}, k_{0}\right),
$$

with the mirror influence function being defined by: 


$$
\begin{aligned}
& I_{\operatorname{mir}}\left(\rho, z ; \omega_{S}, k_{0}\right)=\frac{1}{2 r} \cdot \sum_{L, M=0}^{\infty} \mathrm{e}^{-i(M-L)\left[2 \pi n_{0}-T\left(\omega_{0}-\omega_{S}\right)\right]-(M+L) T \Gamma_{0} / 2} \\
& \times\left\{\frac{1}{\cosh \Lambda_{M}\left(\rho, z ; k_{0}\right) \cosh \Lambda_{L}\left(\rho, z ; k_{0}\right)}\right. \\
& \times\left(\frac{1}{r+z} \cdot\left[1-\frac{2 i \tanh \Lambda_{M}\left(\rho, z ; k_{0}\right)}{k_{0}(r-z)}\right]\left[1+\frac{2 i \tanh \Lambda_{L}\left(\rho, z ; k_{0}\right)}{k_{0}(r-z)}\right]\right. \\
& \left.+\frac{1}{r-z} \cdot\left[1+\frac{2 i \tanh \Lambda_{M}\left(\rho, z ; k_{0}\right)}{k_{0}(r+z)}\right]\left[1-\frac{2 i \tanh \Lambda_{L}\left(\rho, z ; k_{0}\right)}{k_{0}(r+z)}\right]\right) \\
& +\frac{1}{\cosh \Theta_{M-1}\left(\rho, z ; k_{0}\right) \cosh \Theta_{L-1}\left(\rho, z ; k_{0}\right)} \\
& \times\left(\frac{1}{r+z} \cdot\left[1-\frac{2 i \tanh \Theta_{M-1}\left(\rho, z ; k_{0}\right)}{k_{0}(r-z)}\right]\left[1+\frac{2 i \tanh \Theta_{L-1}\left(\rho, z ; k_{0}\right)}{k_{0}(r-z)}\right]\right. \\
& \left.+\frac{1}{r-z} \cdot\left[1+\frac{2 i \tanh \Theta_{M-1}\left(\rho, z ; k_{0}\right)}{k_{0}(r+z)}\right]\left[1-\frac{2 i \tanh \Theta_{L-1}\left(\rho, z ; k_{0}\right)}{k_{0}(r+z)}\right]\right) \\
& +2 \operatorname{Re}\left[\frac{\mathrm{e}^{-i \omega_{S}(r-z) / c}}{\cosh \Lambda_{M}\left(\rho, z ; k_{0}\right) \cosh \Theta_{L-1}\left(\rho, z ; k_{0}\right)}\right. \\
& \times\left(\frac{1}{r+z} \cdot\left[1-\frac{2 i \tanh \Lambda_{M}\left(\rho, z ; k_{0}\right)}{k_{0}(r-z)}\right]\left[1+\frac{2 i \tanh \Theta_{L-1}\left(\rho, z ; k_{0}\right)}{k_{0}(r-z)}\right]\right) \\
& \left.-\frac{1}{r-z} \cdot\left[1+\frac{2 i \tanh \Lambda_{M}\left(\rho, z ; k_{0}\right)}{k_{0}(r+z)}\right]\left[1-\frac{2 i \tanh \Theta_{L-1}\left(\rho, z ; k_{0}\right)}{k_{0}(r+z)}\right]\right)
\end{aligned}
$$

Eqs. (18) and (19) represent the main result of this section.

In the extreme far-field zone in the sense of $\rho \gg f$ Eq. (19) reduces to the well-known free-space result:

$$
I_{\text {free }}(\rho, z)=\left(\frac{\rho}{\rho^{2}+z^{2}}\right)^{2}
$$

so that the spectrum (18) assumes the characteristic positional intensity distribution of dipolar radiation, i.e.:

$$
S_{\text {free }}\left(\mathbf{x}, \omega_{S}\right)=\left(\frac{\omega_{0}^{2} d \rho}{4 \pi \epsilon_{0} c^{2} r^{2}}\right)^{2} \cdot \frac{1}{\left(\omega_{0}-\omega_{S}\right)^{2}+\Gamma_{0}^{2} / 4} .
$$

As a consequence of the rotating wave approximation, in this limit the frequency distribution exhibits a purely Lorentzian lineshape. However, in more general cases which do not correspond to the extreme far-field condition the function $I_{\operatorname{mir}}\left(\rho, z ; \omega_{S}, k_{0}\right)$ is not only position dependent but also depends on the atomic transition frequency $\omega_{0}=k_{0} c$ and on the frequency $\omega_{S}$ at which the spontaneously emitted photon is observed. This additional frequency dependence reveals the influence of the parabolic mirror on the spatial behavior of the atomic photon emission.

The spatial behavior of $I_{\operatorname{mir}}\left(\rho, z ; \omega_{S}=\omega_{0}, k_{0}\right)$ at the atomic resonance frequency is depicted in Fig. 1. Apparently, pronounced interference effects take place in the radiation zone at positions of the photon detector from the radiating two-level system comparable to the focal length of the parabola. Due to the specific structure of the field modes capable of coupling to the atomic dipole oriented along the symmetry axis of the parabolic cavity, the spectrum is exactly zero on the axis of symmetry. However, there is a ring zone around the symmetry axis approximately at $\rho / f \sim 2 / \sqrt{3} \approx 1.15$ at which the spectrum is maximal. Along this ring, the spectrum remains appreciable even at asymptotically large distances from the radiating two-level system. However, in all other directions the probability of detecting a photon diminishes quickly with increasing distance from the radiating two-level system.

The dependence of the spectrum of Eq. (18) on frequency and distance orthogonal to the symmetry axis is depicted in Fig. 2. Characteristic spatial interference patterns originating from the presence of the parabolic cavity are again visible. The contour plot of Fig. 2 indicates a small asymmetry of the frequency dependence with respect to the atomic resonance frequency $\omega_{0}$. To a good degree of approximation this asymmetry can be described by a position-dependent frequency shift $\Delta$ according to the relation: 


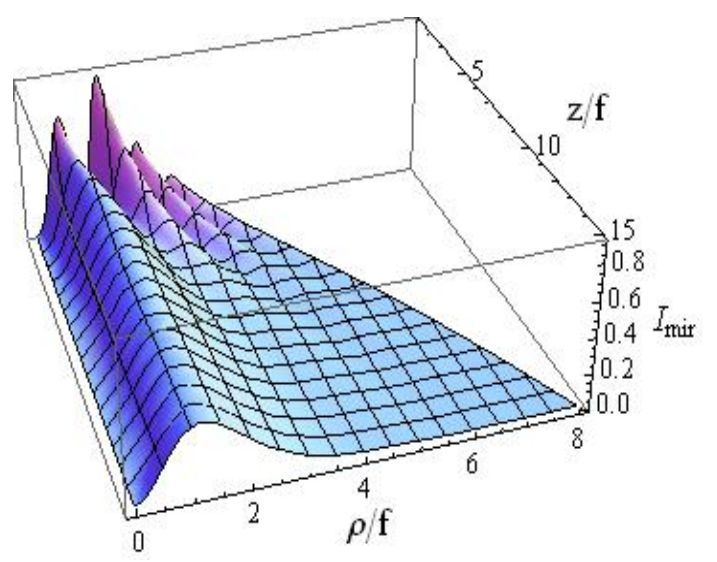

FIG. 1. Position dependence of the mirror influence function $I_{m i r}\left(\rho, z ; \omega_{S}, k_{0}\right)$ as defined by Eq. (19) at frequency $\omega_{S}=\omega_{0}$ for a parabolic mirror. The parameters are $T=20 / \omega_{0}$ and $T=0.1 / \Gamma_{0}$ so that the focal length $f$ is large in comparison to the resonant inverse wave number $k_{0}$, i.e. $f=10 / k_{0}$, and the spontaneously emitted photon is reflected at the boundary of the parabolic mirror many times during time $T$ (compare with Eq. (15)). The boundary of the figure is given by the equation of the parabolic mirror of the form $z / f+1=(\rho / 2 f)^{2}$
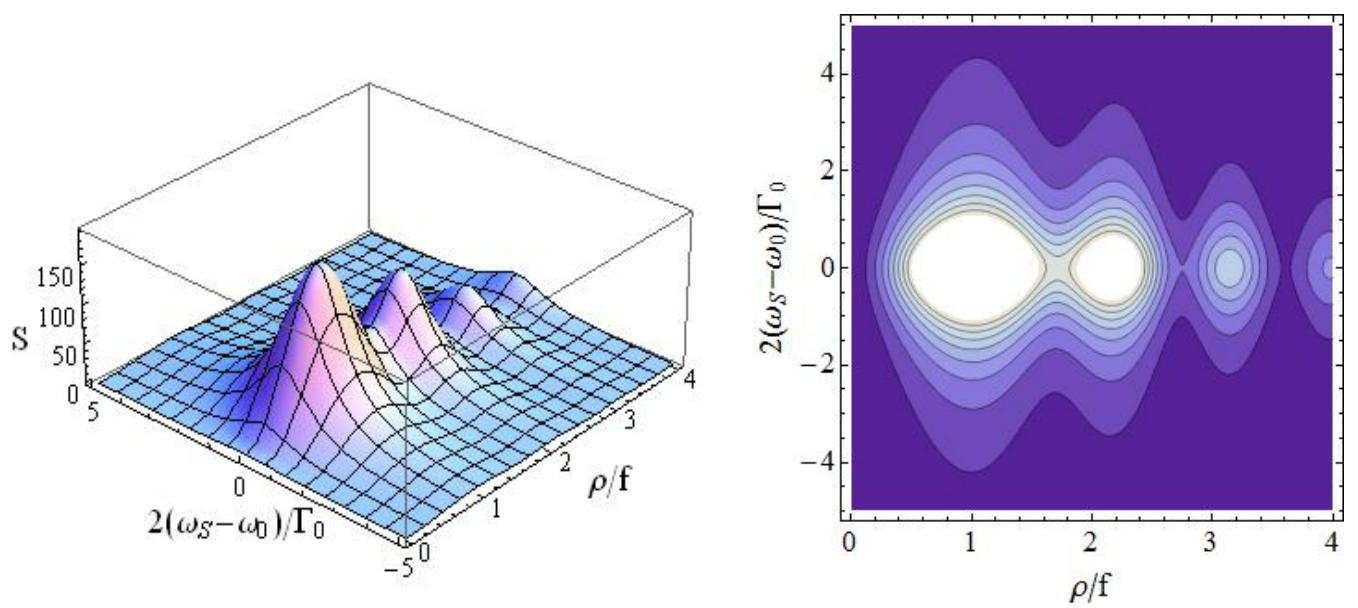

FIG. 2. Dependence of the spectrum of Eq. (18) and of its contour plot on the dimensionless frequency $2\left(\omega_{S}-\omega_{0}\right) / \Gamma_{0}$ and on the distance orthogonal to the symmetry axis at $z / f=3$. The parameters are $\omega_{0} T=20$ and $\Gamma_{0} T=0.1$ as in Fig. 1

$$
S\left(\mathbf{x}, \omega_{S}\right)=\left(\frac{\omega_{0}^{2} d}{4 \pi \epsilon_{0} c^{2}}\right)^{2} \cdot \frac{I_{\operatorname{mir}}\left(\rho, z ; \omega_{0}, k_{0}\right)}{\left(\omega_{0}-\omega_{S}-\Delta\right)^{2}+\Gamma_{0}^{2} / 4},
$$

with the frequency shift $\Delta$ being determined by the equation:

$$
\frac{\partial I_{\text {mir }}\left(\rho, z ; \omega_{0}+\Delta, k_{0}\right) / \partial \omega_{S}}{I_{\operatorname{mir}}\left(\rho, z ; \omega_{0}+\Delta, k_{0}\right)}=\frac{2 \Delta}{\Delta^{2}+\Gamma_{0}^{2} / 4} .
$$

In Fig. 3, the spatial dependence of this frequency shift $\Delta$ is depicted. The modifications of the spectrum originating from the presence of the parabolic cavity give rise to an oscillatory spatial dependence of this frequency shift which reflects once again characteristic interference effects taking place inside the parabolic cavity.

In order to demonstrate the accuracy of the approximation of Eqs. (21) and (22) a cut of Fig. 2 at position $\rho / f=0.5, z / f=3$ is depicted in detail in Fig. 4 (left) with its top part being amplified in Fig. 4 (right). Although this shift is small, i.e. $2 \Delta / \Gamma_{0} \approx-0.003$, it is clearly visible. 


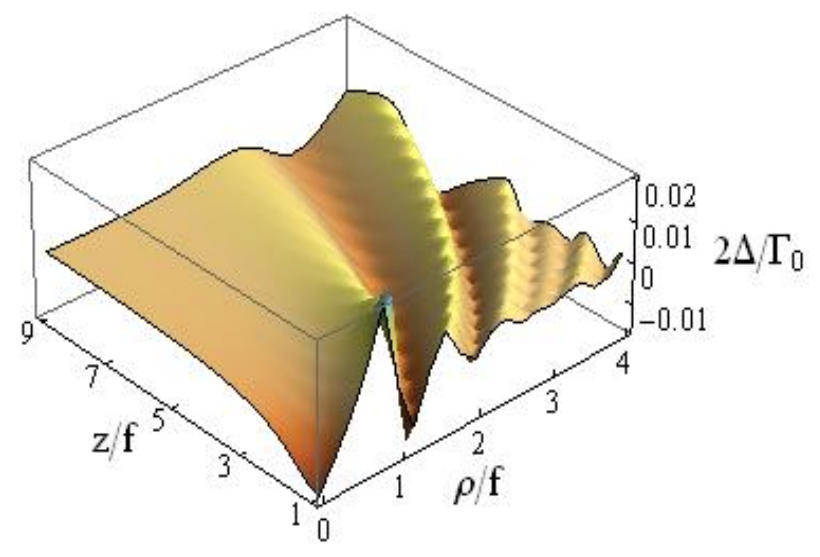

FIG. 3. Position dependence of the effective shift $2 \Delta / \Gamma_{0}$ as defined by Eqs. (21) and (22). The parameters are $\omega_{0} T=20$ and $\Gamma_{0} T=0.1$ as in Fig. 1
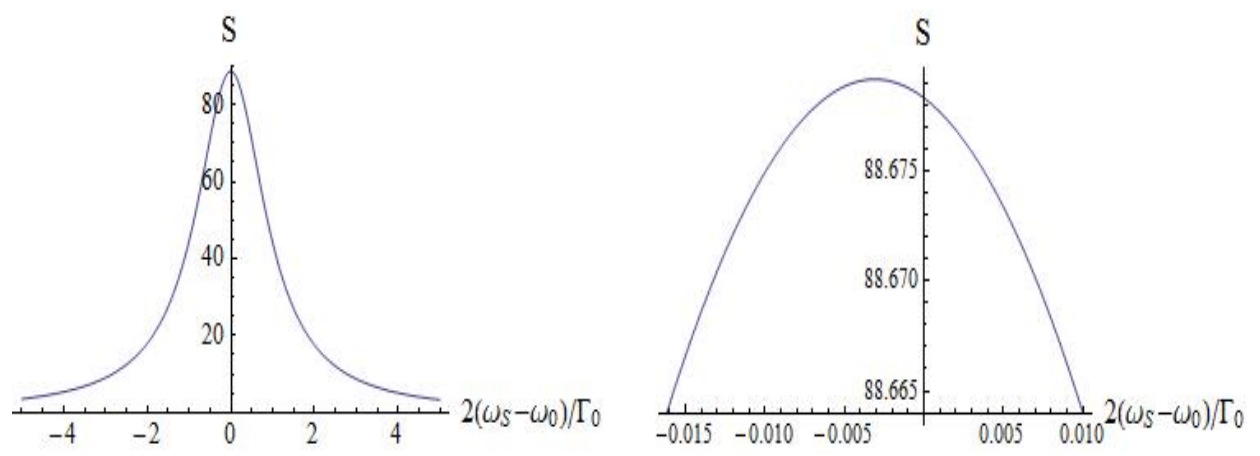

FIG. 4. Spectrum of Fig. 2 and its dependence on the dimensionless spectral frequency $2\left(\omega_{S}-\right.$ $\left.\omega_{0}\right) / \Gamma_{0}$ at the fixed position $\rho / f=0.5$ and $z / f=3$. The parameters are $\omega_{0} T=20$ and $\Gamma_{0} T=0.1$ as in Fig. 1. The right part of this figure shows the frequency dependence around the maximum on a finer scale

\section{Summary and conclusion}

We have investigated the spectral properties of a photon spontaneously emitted by a motionless two-level atom positioned at the focus of a parabolic cavity with a dipole moment oriented along the symmetry axis of the parabola. Within a quantum optical model we have studied the spectrum of the spontaneously emitted radiation with the help of a photon path representation of the first-order electric field correlation function. In this manner, the influence of the parabolic mirror on the spontaneous photon emission process is described in terms of photon path contributions originating from repeated reflections of the spontaneously emitted photon at the boundary of the parabolic mirror. It has been demonstrated that inside the parabolic cavity, the position and frequency dependence of the spectrum of the spontaneously emitted photon exhibits interesting interference patterns. In the radiation zone, the spatial behavior of the spectrum reveals strong interference at distances from the atom of the order of the focal length of the parabola. With increasing distances, these interference patterns decay in all directions except for an undepleted component along the axis of symmetry. Furthermore, the maximum of the spectral line-shape exhibits a position-dependent frequency shift with respect to the atomic resonance frequency.

\section{Acknowledgements}

This work is supported by the Heisenberg-Landau Program (JINR, Russia). 


\section{References}

[1] Bruss D., Leuchs G. Lectures on Quantum Information. Wiley-VCH, Weinheim, 2008.

[2] Haroche S., Raimond J.M. Exploring the Quantum: Atoms, Cavities and Photons. Oxford UP, Oxford, 2006.

[3] Jaynes E.T., Cummings F.W. Comparison of quantum and semiclassical radiation theories with application to the beam maser. Proceedings of the IEEE, 1963, 51 (1), P. 89-109.

[4] Paul H. Induzierte Emission bei starker Einstrahlung. Annalen der Physik, 1963, 466 (7-8), P. 411-412.

[5] Schleich W.P. Quantum Optics in Phase Space. Wiley-VCH, Weinheim, 2001.

[6] Maiwald R., Golla A., et al. Collecting more than half the fluorescence photons from a single ion. Phys. Rev. A, 2012, 86 (4), $043431-5$.

[7] Trautmann N., Bernad J.Z., et al. Generation of entangled matter qubits in two opposing parabolic mirrors. Phys. Rev. A, 2014, 90 (6), 063814-9.

[8] Purcell E.M. Spontaneous emission probabilities at radio frequencies. Phys. Rev., 1946, 69 (8), P. 681.

[9] Alber G. Photon wave packets and spontaneous decay in a cavity. Phys. Rev. A, 1992, 46 (9), R5338-R5341.

[10] Alber G., Bernad J.Z., et al. QED with a parabolic mirror. Phys. Rev. A, 2013, 88 (2), 023825-11.

[11] Abramowitz M., Stegun I. A. Handbook of Mathematical Functions. Dover, New York, 1965.

[12] Eberly J.H., Wódkiewicz K. The time-dependent physical spectrum of light. J. Opt. Soc. Am., 1977, 67 (12), P. 1252-1261. 